\title{
Travel Behaviour and Society
}

Volume 17, October 2019, Pages 26-35

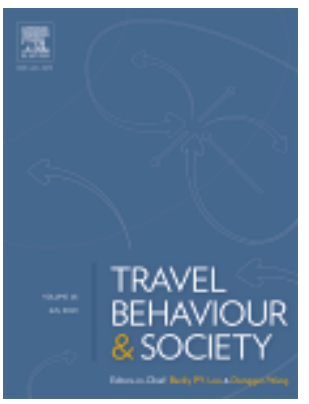

Qualitatively exploring the effect of change in the residential environment on travel behaviour

Author links open overlay panelTemitopeFarinloye ${ }^{a}$ EmmanuelMogaji ${ }^{b}$ StellaAririguzoh ${ }^{\mathrm{T} T a i} \mathrm{AnhKieu}^{\mathrm{d}}$

Show more

https://doi.org/10.1016/j.tbs.2019.06.001Get rights and content

\section{Abstract}

Qualitative research with residents relocating from London was undertaken to develop an understanding of how and to what extent a change in the residential environment affected people's travel behaviour and attitudes. Data was collected through semi-structured interviews and was thematically analysed. The findings reveal that when in a new location, residents observe the features of their built environment, identify the cause of their stress, and make efforts to address it with a change in travel behaviour. The key contribution of this study is the realisation of different levels of travel behaviour in response to a change in residential location - some residents maintained their travel behaviour, some complemented it, while some changed their behaviour to adapt to their new built environment. Theoretically, this research contributes to the extension of knowledge on travel behaviour as it focuses on suburbanising Londoners; the qualitative method adopted for this research also contributes to current knowledge. Practically, there is the potential of developing a travel behaviour change initiative around ridesharing and policy changes and initiatives to improve physical planning and sustainable travel.

- Previous article in issue

- $\quad$ Next article in issue

\section{Keywords}

Travel

Relocation

London

Behaviour

Residential self-selection 
Qualitative research 